Meta

Journal des traducteurs

Translators' Journal

\title{
Cette sagesse qui bouscule
}

\section{Stéphane Bédard}

Volume 40, numéro 4, décembre 1995

URI : https://id.erudit.org/iderudit/002012ar

DOI : https://doi.org/10.7202/002012ar

Aller au sommaire du numéro

\section{Éditeur(s)}

Les Presses de l'Université de Montréal

ISSN

0026-0452 (imprimé)

1492-1421 (numérique)

Découvrir la revue

Citer cet article

Bédard, S. (1995). Cette sagesse qui bouscule. Meta, 40(4), 564-578.

https://doi.org/10.7202/002012ar

\section{Résumé de l'article}

La transmission du discours de sagesse joue dans l'oralité. Comment traduire de l'anglais au français un message porté par une voix? La tradition française, fortement axée sur l'écrit, tend à faire taire la voix de l'auteur et donc la voie traduite : elle vise souvent à la ramener à une construction sentie comme littéraire, amie du beau et de la clarté. L'ethnocentrisme perdure, ferment d'exotisation. Qu'advient-il alors de la signifiance du texte comme le signale Meschonnic? Comment réduire la dualité entre le discours traduit et son contenu? La connaissance de soi et des habitudes bien ancrées du traducteur - issu d'une société éprise de normalisation - est proposée comme moyen d'échapper au dogme à la mode : l'hyperanalyse du texte
Ce document est protégé par la loi sur le droit d'auteur. L'utilisation des services d'Érudit (y compris la reproduction) est assujettie à sa politique d'utilisation que vous pouvez consulter en ligne.

https://apropos.erudit.org/fr/usagers/politique-dutilisation/ 


\title{
CETTE SAGESSE QUI BOUSCULE
}

\author{
STÉPHANE BÉDARL

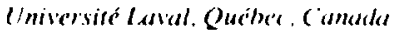

\begin{abstract}
Résumé
La transmission du discours de sagesse' se joue dans l'oralité. Comment traduire de

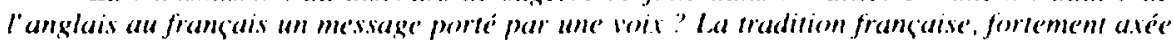

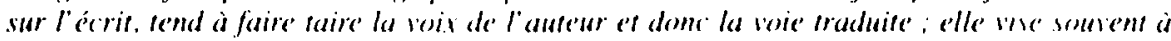
la ramener à une construction sentie comme littéraire. amie du beau ef de la clarte' l'othmo-

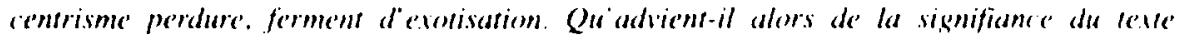

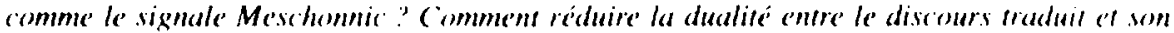

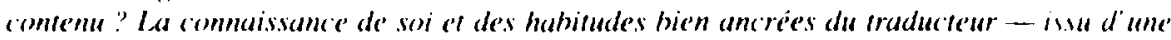
société éprise de nomalisation - e'st propessée comme moyen d'échapper au desme à la mode : l'hyperanaluse du tevts
\end{abstract}

\begin{abstract}
The transmission of wisdom in discourse is a phenomenon of orality. How deses ane translate an oral message from English into French." The French tradition, which contres around writing, tends to silence the author's boice. and consequents, the mode of expression. and sceks of transform it into a literary-like construction, blending beauty and clarits: Ethnocentrism is unrelenting as combeism forments. But. as Meschomnic narns, what is to hecome of teat meaning? Hen can one best deal with the dichotomy hetueen translated discourse and its content.' The author suggests that the translator - horn in a nerm-conscrious socie'ty - acquire self-knenledge and le'drn to undersfand his or he' own deeply ingrained hathits as a means of escaping the dogma of the day name's text hyperanalysis.
\end{abstract}

\section{SUR LE TANTRA DU TIBET}

Tout le discours du lantra est l'expression, sinon la proclamation directe et intrépide d'un état de complétude achevé, un état dit de "présence pure et totale», accessible à tout être. Lorsqu il est authentique. ce discours, caractérisé par sa spontanéité, est proclamé par un maître en contact primordial avec la «réalité telle qu'elle est». c'est-à-dire exempte de fabrications conceptuelles. Cet acteur de l'éveil doit manifester concrètement en actes et en paroles qu'il a ćtabli un rapport direct ét intime avec le monde phénoménal. Lorsque le maître tantrique utilise la parole et la langue pour exprimer l'expérience de la "présence pure et totale». la tradition bouddhique dit de lui qu il s inscrit dans la "lignéc de la transmission orale".

La parole d'un maitre du tantra, peu importe la forme qu'elle prenne (poésie. liturgie, causerie, texte) est un acte qui se jouc dans l'instantanéité. dans l'ici et le maintenant. Il y a prise de parole, offrande de parole parce que, et seulement parce que, des enseignements sont demandés par un élève ou un groupe d'élèves. Ce discours de spontanéité est exempt de frivolité, de verbiage, de papotages intellectuels, de tergiversations. Il arrive que cette parole soit signifiante au point où l'on en réclame la traduction dans de nombreuses langues. C"est le cas du discours tantrique de Trungpa. En cette fin de siècle hyper agitée. la Terre et les hommes s'essoufflent. Certains d'entre eux veulent lire et entendre une parole dont ils auront à juger de la teneur en sagesse. Pour le traducteur ayant le privilège de rendre ces textes accessibles à un vaste public, il y a lieu de poser la question : de quoi ai-je besoin pour traduire un tel discours? Quelles sont les conditions 
préalables à la production d'une traduction rendant compte d'une expérience de la "présence pure et totale» au monde ? pureté ? totalité ?

Que le lecteur se rassure, je ne me propose pas de l'entretenir ici de cette nouvelle mode qu'il est convenu d'appeler concept de la «qualité totale». L'idéalisme cadre mal avec le tantra et la traduction tout court. L'entreprise à laquelle je le convie est plus terrea-terre, pragmatique et ancienne. La traduction du discours bouddhique du sanskrit au tibétain remonte. en effet, à l'année 779 . Bien que vénérable, la lignée de la traduction est maintenant éteinte au Tibet pour au moins deux bonnes raisons : tous les textes d'importance ont été traduits et l'envahisseur actuel - la Chine - conformément au dogme communisant, ne prise guère les travaux faisant état de la possibilité pour tous les êtres doués de sensibilité de connaître l'éveil individuel. La Chine, pour l'heure, croit fermement à l'éveil "collectif" qu'elle fait payer cher aux Tibétains : rien de moins que le génocide systématique.

Cet état de choses n'a heureusement pas que des effets désastreux. Le Nobel de la paix remis au Dalai Lama en 1989 l'aura tout au moins aidé à sensibiliser l'opinion internationale au drame qui afflige ses compatriotes, à émouvoir de sa douceur légendaire un Occident anxieux. En outre, depuis plusieurs décennies, des maîtres tibétains forcés à l'exil pour cause de barbarie se sont réfugiés en Inde, au Népal ou en Occident. Ils ont commencé à enseigner aux Occidentaux ; un nouveau cycle de traduction du tibétain vers diverses langues modernes commence à peine. Certains de ces maîtres enseignent directement en anglais. Chögyam Trungpa, dont j'étudie et traduis l'œuvre depuis une quinzaine d'années, est l'un d'eux. Son rayonnement dans la francophonie est déjà considérable puisque neuf de ses ouvrages ont déjà paru aux Éditions du Seuil, dont huit dans la collection Sagesses.

Même lorsqu“il traduit de l'anglais un discours de sagesse réputé asiatique. Ie traducteur occidental a toutefois intérêt à méditer cette observation de Steiner :

Erroneously or not, by virtue of initial chance or method, the Western eye fixed on certain constants - or what are taken to be constants - of Chinese landscape, attitude, and emotional register. Each translation in turn appears to corroborate what is fundamentally a Westem invention of China. (1977:359)

Quels sont donc les préalables à respecter? Toujours les mêmes : très bien connaître les langues de départ et d'arrivée, avoir une excellente compréhension du domaine à traduire et maîtriser les techniques de traduction. Dans le cas du bouddhisme, une autre exigence se révèle essentielle. En effet, la connaissance théorique du tantra n'est pas suffisante. Le traducteur doit avoir fait l'expérience personnelle, même si elle n'est que partielle, du discours à traduire, sans quoi tout l'implicite des enseignements à transmettre risque d'être complètement occulté. De plus, pour trouver le "ton", la «couleur», la «musique», le «rythme», la "lessiture» même de cette parole - dont il faut bien admettre qu'elle est inusitée en Occident —, il faudra recueillir le plus d'éléments possible sur la «vie» de leur auteur et les méthodes très singulières qu'il employait pour rendre compte de son expérience.

\section{TRADUCTION ET TRANSMISSION ORALE}

Le discours oral impose el confirme une "présence». Pour la transmission orale du discours de sagesse cela signifie que corps et esprit chez la personne qui parle sont synchrones. Cette présence marquée par la parole, son organicité, sa spontanéité, son immédiateté, enrichit le sens. C'est notamment par l'oralité et le rythme qu'elle crée que des sens sont constamment transmis et débordés. La voix installe le discours à un niveau qui dépasse celui que posent ultérieurement sur papier des transcripteurs ou copistes. Dans ce 
passage de l'oral à l'écrit, il est inévitable que des pertes se produisent; il s'agit de se demander si la traduction - comme acte de réécriture - peut contribuer à réduire l'ampleur de la perte et comment.

\section{POUVOIR DE L.ORALITE.}

Dès 1972. Chögyam Trungpa tenait deux séminaires portant sur la folle sagesse, sujet qu'il qualifiait lui-même d'une extraordinaire difficulté : «Certains d'entre vous seront peut-être complètement désorientés. $D^{\prime}$ autres pourront très bien en tirer quelque chose $[\ldots]$ C'est là un sujet d'une extrême subtilité, et certains de ses aspects sont très difficiles à décrire." (1993: 13) Chacun des séminaires durait à peu près une semaine. La plupart de ceux qui y participaient n'étudiaient le bouddhisme que depuis 1970. Pourquoi présenter une matière aussi ardue à des débutants? Et, dans ces conditions, comment procéder pour les faire rester sur place pendant une semaine? Cette tactique de Trungpa était fréquente. Les étudiants qui sortaient de ces séminaires avaient souvent l'impression de n'y avoir rien compris, mais curieusement ils étaient, dans l'ensemble, à la fois étonnés et enchantés d'avoir pu rester "présents» même s’ils n'avaient pas saisi une grande partie des enseignements donnés. Que se passait-il donc lors de ces rencontres pour que des adultes intelligents restent là à ne rien comprendre ? Qu'est-ce qui retenait leur altention ? On peut supposer que c'était la présence d'un être qui incarnait son discours avec une voix disséminant des sens multiples, insondables, à un rythme qui ouvrait sur un espace hors du rationnel. Cette présence était l'enseignement qui, par définition, est indicible. En 1991, ces deux séminaires ont été publiés sous le titre Crazy Wisdom. Dans sa préface, la personne chargée de préparer ce texte pour la publication remarque : «Un grand effort a été fait pour ne pas travestir le langage de Trungpa Rinpoché ni modifier son style à seule fin d'obtenir un ton plus conventionnel, plus présentable.» (1993:9)

Ce climat instauré par une voix influence la traduction. et notamment la traduction du rythme propre au discours de Trungpa. Meschonnic:

Je définis le rythme dans le langage comme l'organisation des marques par lesquelles les signifiants. linguistiques el extralinguistiques (dans le cas de la communication orale surtout), preduisent une sémantique spécilique, distincte du sens lexical. et que j’appelle la signifiance : c'est-à-dire les valeurs propres à un discours et à un seul. (1982:216-217)

lci, le rythme a valeur de sens : l'air compte tout comme les paroles. L'air et les silences autour, les soupirs, les sourires, les gestes. les regards. l'expression faciale sont autant de composantes du discours transmis oralement. Et comme voix et parole sassocient pour signifier, qu'advient-il alors du sens dans le texte écrit. qui a forcément perdu la voix ?

Le traducteur a-t-il raison de s'acharner à rendre sinon la voix, au moins son rythme, son énergie vitale ? Derrida rappelle :

Le ton et la force signifient la vesis présente : ils sont antéricurs au concept. ils sont singuliers. et ils sont d'autre part altachés aux voyelles, a l’élément vocal et non consonantique de la langue. La force d'expression ne revient qu' au son vecalique, au moment où le sujet est la. en personne, pour proférer sa passion. Quand le sujet n'est plus là, la force, l'intonation. l'accent se perdent dans le concept. Alors on ecrit. on supplée en vain à l'accent par . les accents», on se soumet à la généralité de la loi. |... La phrase parlée, qui ne vaut qu une fors el reste "propre seulement au lieu où elle est", perd son lieu et son sens propre dés qu elle est écrite. (1967:44.3)

Une telle affirmation semble vouer à l'échec la traduction d'enseignements oraux. Est-ce a dire qu'on ne pourra en traduire que les signifiés, jamais la signifiance ? Ce qui porte à croire que traduire l'oral, c'est favoriser d'emblée le glissement de sens. On se voit donc 
forcé de remettre en cause les ressources du discours et de ceux qui l'écrivent ou le traduisent. Pourtant, des textes bouddhiques ont été traduits depuis des centaines d'années : le message continue d'être transmis de génération en génération.

\section{TRADITION dE L.A PAROLE. ET TANTRA}

C'est précisément grâce à la tradition orale selon laquelle les instructions sont transmises de maître à élève que les enseignements tantriques du Tibet ont été préservés jusqu'à nos jours. Parallèlement à cette tradition de la parole, des travaux de traduction gigantesques, dans de nombreuses langues asiatiques, ont marqué toute l'histoire du bouddhisme. Maintenant que ce mode de pensée s'enracine en Occident, les moyens techniques dont nous disposons pour faire entendre cette parole changent toute la perspective de sa traduction dans les langues occidentales. Dans le cas des enseignements dispensés par Trungpa, nous avons accès à des archives sur bandes magnétiques très nombreuses qui sont autant d'outils pour le traducteur. En outre, plusieurs documents vidéo tournés à l'occasion de séminaires permettent désormais au traducteur de modifier son approche du texte et de la parole justement parce que paroles et images ont été consignées sur support magnétique. Dans ces conditions, l'excuse selon laquelle la voix meurt dans l'écrit perd de sa force. Le traducteur ayant accès à la voix peut en entendre les rythmes et mouvements et s'en inspirer pour accomplir sa tâche.

Les traducteurs qui n'ont pas eu la chance d'étudier auprès de Trungpa eux-mêmes peuvent donc entendre son discours. Et le traducteur qui fait fi de ces outils que nous offre l'ère de l'électronique risque gros : ses lecteurs aussi ont accès aux cassettes audio et vidéo. Ce qui explique pourquoi il est désormais périlleux d'occulter le caractère oral dans les textes à traduire puisqu'il est une composante essentielle de leur sens, toujours a notre portée. Ajoutons que les textes des maîtres sont souvent lus a haute voix en salle de méditation : la parole est sans cesse réentendue. Pour demeurer authentique, elle devra donc, entre autres, bousculer, au besoin, les règles de l'écrit.

\section{I.A L.ANGUE DU BOUDDHISME}

Dans son Histoire du Bouddhisme indien, Lamotte nous apprend que l'usage de la langue pour exprimer la parole du Bouddha historique a soulevé de nombreux débats, qui auraient abouti, selon lui, aux conclusions suivantes:

Tout bien pesé, il semble que le Buddha $\mid$ sic $\mid$ interdit de psalmodier les textes bouddhiques avec les intonations en usage dans la récitation des Veda', mais ordonne que chaque disciple enseigne la parole du Buddha dans son dialecte propre. |... Il ne faut pas s'en tenir à un vocable unique. sous prétexte qu'il est le seul correct. mais adopter le vocable en usage dans la région où l'on se trouve. $(1976: 611)$

En outre, ces paroles du Bouddha sont autant d'indications précises sur la manière dont il désirait qu'on aborde son discours : «Dans ma religion, il ne s'agit pas de beau langage (mei-yen). Que le sens et le raisonnement ne soient pas déficients, voilà tout ce que je veux. Il faut prêcher selon les sons qui permettent aux êtres de comprendre. C'est pourquoi il est dit qu'il faut se comporter selon les pays." (1976: 612) À première vue. on peut penser que le Bouddha prône une approche platonicienne du discours étant donné qu'il en privilégie le sens, bien que, dans le même souffle, il semble donner raison à Nida dont toute la théorie sur la traduction est fondée sur l'adaptation au destinataire du texte.

De manière générale, on peut dire que les bouddhistes étaient libres d'employer la langue qu'ils préféraient et que «le choix du parler dépendait en fin de compte d'habitudes invétérées ou de simples raisons d'opportunité». (1976:614) On ne semble donc 
pas connaître de «registre sacré" qui s’appliquerait à la diffusion de la parole d'un houddha. En outre, on dit qu'une des caractéristiques singulières des maîtres bouddhistes accomplis est bien celle de rendre compte de leur expérience d'une manière simple et directe, sans s’embarrasser d'un jargon spécialisé ni de raisonnements métaphysiques abscons. Le tantra se caractérise notamment par son penchant pour traduire l'expérience immédiate de l'éveil. Cet éveil est proclamé, proféré, incarné sur-le-champ dans le discours du maître.

Pourquoi vouloir conserver au discours sa signifiance? Ce type de question commence à préoccuper de plus en plus les ethnologues qui sont aux prises avec les problèmes du passage de l'oral à l'écrit: al'ethnologue, porte-paroles et porte-plumes. textualise le réel el court le risque d'abstraire d'emblée de son champ d'observation des dimensions relevant d'une autre logique que celle de l'écriture». (Schlechten 1987: VII) Il est clair, et tous les réviseurs du texte de Trungpa le signalent, qu on ne peut dissocier totalement la forme du message de son contenu. La forme n'est pas innocente : ce serait faire violence au discours que de l'occulter. Fi cette forme du discours propose un autre ordre de signification. Mais des considérations pratiques entrent en ligne de compte. Nous semblons pressés d'arrêter le sens, comme nous le verrons plus loin, pour le rendre lisible, consommable. La production du texte et de la traduction n'est-elle pas des lors une dérive ? Quels sont les effets de celle dérive sur le lecteur? Quelles sont lé limiter a ne pas franchir?

\section{F TEXTE : ENONCE OU ENONCIATION}

Ce sont notamment les travaux d'Antoine Berman et d'Henri Meschonnic qui mont amené à m'interroger sur la justesse des traductions des enseignements de Trungpa, el sur ses difficultés spécifiques. Meschonnic critique les traductions françaises de la Bible dont il dit qu elles "passent diversement à l'écrit désoralisé". (198.5: 18) Il s'élève contre l'approche herméneutique de la traduction qui a opéré un clivage entre forme et sens, langue el discours, et $n$ 'a choisi d'en traduire que le sens lexical et syntaxique. Contre ce dualisme de la traduction, il milite en faveur d'une pótique du traduire qui mettrait en valeur le texte comme système : "ainsi le discours est de lordre du continu. La langue, avec ses unités, est de l'ordre du discontinu. Les distorsions à l'usage, šil y en a, sont les éléments de ce système et de ce continu du discours. Alors seulement il y a une poétique du traduire. comme il y a une poétique de l'écriture". ( 1986 : 79) Celte théorie revendique pour le traducteur une pratique d’ècriture. Pour écrire, réécrire, il devra traduire le discours et non la langue. Le texte est vu comme système de signifiance et non comme accumulation de phrases auxquelles on devra trouver des équivalents justes. À la question qu est-ce que l'exactitude. Meschonnic répond :

L'exactitude n’est que du sens. ("est-à-dire seulement une partie du sens. Puisqu elle oublic que le sens ña lui-même qu'une part dans le mode de signifier. Même dans un texte philosophique. De Platon à Hegel, les philosophes n'ont cessé de jouer avec les mots. Avec lcur langue. (1986: 78)

Dans cette perspective, le texle traduit pourra donc transformer la langue à cause du discours et s'écarter des présupposés esthétisants pour rassurer le lecteur, lui redonner sès habitudes de heauté.

Antoine Berman, pour sa part, pose le problème du lien étroit entre philosophie et traduction. Il insiste pour dire que ses travaux sont le fruit d'une expérience de la traduction et d'une réflexion sur celle-ci. et non l'ébauche d'une théorie. Dans le même esprit que Meschonnic, il écrit : «la traduction trouve sa force propre a écarter le "signifiant" pour capter le "signifié". Car on ne traduit pas des "mots" mais du "sens". Proposition rebattue, qu'on enseigne encore dans toutes les écoles de traduction $|\ldots|$ " 
(1986: 64) Cette approche de la traduction correspond pour lui à la pensée platonicienne selon laquelle l'idéal consiste à restituer le sens. S'oppose à cette vision de la traduction la méthode cartésienne qui vise avant tout la clarté de l'énoncé, et la manière de l'idéalisme allemand voulant que le texte traduit renferme tout le pouvoir du texte de départ.

Les réflexions de Berman indiquent avec finesse que souvent la traduction pallie son manque d'être une écriture par un surcroît de sens. Le texte traduit est énoncé et non énonciation. Le traducteur, parfois pris de déséquilibre devant le texte étranger et son silence, supplée par une parole nerveuse. qui le sécurise : il embellit le texte de départ pour ne pas tout perdre. Berman a bien observé cette peur :

Mais il se produit le plus souvent ceci : dès que le traducteur a découvert les points faibles du texte. il s'empresse de jeter sur eux le voile du sens et de l'être : il fait apparaitre les beautés. et disparaître les défauts. Le platonisme de la traduction confine ici a la rhétorique sophistiquée. (1986: 71)

Che 2 beaucoup de lecteurs, la beaute formelle est perçue comme un signe de qualité. En règle générale, on ne remet pas en question un texte qui se lit bien : on dit alors qu'il coule de source. Quel lecteur, quel éditeur même, remonte au texte de départ pour vérifier la justesse d'une traduction qui se lit si bien? En matière de traduction, l'expérience a montré qu'on se fie aux apparences. Ici, le mot clef c'est la «transparence". Ce qui veut souvent dire traduire comme si les traces du discours et de son auteur, c’est-à-dire de l'autre, devaient être annulées. Faire taire les signes de la présence "autre" pour que règne l'ordre ambiant. On demande souvent au traducteur de ne pas déranger, de ne pas se prendre pour un auteur. Mais en quoi ces considérations théoriques intéressent-elles la traduction du discours de sagesse?

\section{I.'KTHNOCENTRISME: ET SES MULTIPLES VISAGES}

Dans un article sur la traduction des auvres latino-américaines, Berman fait remarquer que :

Partout où la traduction française est confrontée à des auvres où l'oralifé est présente, qu'il s'agisse de la Bible, de Shakespeare ou des romanciers d'Amérique du Sud, elle se heurte immédiatement aux tendances antivernaculaires de notre langue littéraire. Tendances qui constituent un swssème de déformation dont il faut prendre une conscience elle-même systématique. ( $1982: 39-40)$

En étudiant divers extraits de traductions de l'auvre de Trungpa, on peut facilement repérer bon nombre de ces tendances inconscientes.

Le dilemme qui oppose les diverses écoles de traduction depuis des siècles refait toujours surface quel que soit le domaine de traduction. La lettre ou l'esprit? Le sens avec ou sans la signifiance? Trungpa semble proposer pour sa part de ne pas trop "solidifier» les positions. Comme si le traducteur devait se faufiler entre les tendances à la mode ou démodées. Il n'en reste pas moins que celui-ci est marqué par sa culture d'origine. Ce n'est pas un pur esprit à l’abri des présupposés chers a la société où il vit. Pour travailler avec lucidité, il doit critiquer son travail, s’interroger sur ses partis pris. À ce sujet, Barthes signale :

La culture française a toujours attaché, semble-t-il, un privilège très fort aux «idées», ou, pour parler d'une façon plus neutre, au contenu des messages. Importe au Français le "quelque chose à dire». ce qu'on désigne couramment d'un mot phoniquement ambigu. monétaire, commercial et littéraire: le fond (ou le fonds ou les fonds). |... L La culture française n'a connu pendant des siecles que le travail du style. les contraintes de la 
rhétorique aristolo-jésuite. les valeurs du «bien-écrire», elles-mêmes centrées, d’ailleurs. par un retour obstiné. sur la transparence el la distinction du «fond». (1984:265)

Le traducteur imprégné de celle culture et de la littérature qu elle produit aura tendance à accumuler des habitudes de déformation influant sur les textes qu'il traduit de sorte que sans s'en rendre compte, aveuglé par sa difficile tâche, il perpétue l'idéologie de l'opposition, entre énoncé et énonciation, sens et langue, fond et forme, signifiant et signifié.

Pour passer à un exemple concret. voici un court extrait d'un texte de Trungpa dont l'examen est assez révélateur dans la mesure où il renferme un nombre respectable de gaucheries et d'erreurs. L'intérêt de ce texte, c'est qu'il montre le travail d'un apprenti traducteur qui n'a même pas encore assez d'adresse pour masquer ses penchants déformants. L'original de cet article a été publié dans une revue universitaire: Tho Naropa Institute Journal of Psychology. L'article est adapté d'une causerie donnéc a l'occasion d'un colloque de psychologie à ce même institut.

Voici la traduction française du dernier paragraphe de cet article :

Un point crucial est. évidemment, de ne pas cautionner la folie des gens, hien que nous puissions par ailleurs les apprécier. Nous me ponvons approuser quelque sorte de folic que ce soit. si légère soit-elle. ê même pas la nôtre. Nous n'avons pas à faire mombe d'une poigne de fer, mais comme nous l'avons dit, nous pouvons travailler ave' tout rapport que quelqu' un puisse aroir, aver chaque perite chesse simple. Il s'axit de travailler arec le poim

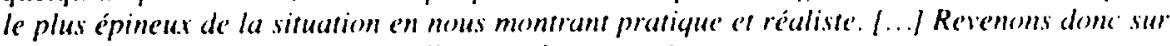
terre : nous pourroms alors travailler arec le's autres.:

Plusieurs éléments surprennent ici : le ton enfantin (étrange dans un article destiné à des universitaires) : la cascade de «nous» qui rend le discours tout à fait artificiel - à noter qu'en début d'article, on indique qu'il s'agit de l'adaptation d'une causerie : le charabia de la troisième phrase : et le ton impératif, voire puéril, d'un article qui par ailleurs s'étire sur 7 pages. Voici le texte anglais :

One hasic point is, obviousls, not to go along with people's craziness, although wo appreciate them. You can'l go along with craziness of any kind at all. cren the slightest bit. e'ten your own crasiness. You do not have to be heary-handed, particularly. but as we have said. you can work with whatever commection a person may have, any little simple thing. Try to nork with the pinpoim of the sitwation by being very practical and ordinary. /.. / So we have to come down to earth. The'n we can wark with others.'

Ce court passage, et l'ensemble de cette traduction, du reste, est un parfait exemple de traduction bouddhique à saveur ethnocentrique. Examinons ce travail étrange point par point. La première phrase est un mélange de mot à mot et d’amplification. En outre, le verbe acautionner" fait passer le discours à un autre registre. Le traducleur ajoute un subjonctif inutile et casse la logique de la phrase en la rationalisant pour en déformer le sens et l'allonger. Il détruit l'oral pour faire écrit. Tout le système des nows alourdit le texte et le désoralise. Avec la deuxième phrase. le ton du texte anglais est occulté : le caractère intimiste, simple, parlé saute - l'énonciation devient énoncé. Dans la phrase suivante, le traducteur senlise au point de faire du charabia : que signific. en effet. «travailler avec tout rapport que quelqu un puisse avoir»? $\dot{A}$ noter, que dans la phrase qui suit Trungpa donne un conseil (Try (o), que le traducteur escamote complètement en rendant cette phrase par une affirmation. Ce qui constitue un détournement de sens. salns parler des deux faux sens qui «achèvent» cette phrase : «pinpoint" n'est pas un point épincux et "ordinary", terme technique dans la langue de Trungpa, n'a jamais voulu dire "réaliste". La dernière phrase de l'article résonne un peu comme un slogan scout. Ici l'atltéur 
est infantilisé, il termine son texte par un commandement aux accents néocuristes bouddhisant.

En somme. le traducteur de cet article nous offre un exemple parfait des dangers de la rationalisation, qui modifie les structures syntaxiques du texte de départ au point de construire un nouveau texte ayant peu à voir avec le discours d'origine. Par le fait même. il rend un texte clair plus abstrait. Comme il comprend mal cet article, il a tendance a le clarifier pour le lecteur : l'ennui, c'est que pour rendre plus explicite il faut d'abord avoir saisi. Il est évident que notre traducteur entend mal l'anglais. Comme il a du mal à se dégager du texte de départ, et qu'il veut produire de la clarté, il se voit obligé d’allonger. C'est ainsi qu'il arrive à déborder, à donner des sens qui ne sont pas dans l'article original. L'emploi des subjonctifs, par ailleurs, fait de ce discours oral une rhétorique aux accents vieillots. Trungpa s'y exprime en français parfois comme un boy-scout - appauvrissement qualitatif du discours - tandis qu'ailleurs il a l'air de faire un prêche. Les erreurs de terminologie et de sens appauvrissent d'autant le texte. Comme le traducteur hésite constamment entre la traduction servile et la reconstruction systématique du texte, il est évident qu'il ne peut insuffler un rythme organique à son texte, qui fonctionne dès lors par à-coups, phrase par phrase, sens par sens : des idées sont juxtaposées sans grand effort de systématisation ni d'écriture. En fait, il opère le plus souvent un transcodage qui le force, à loccasion, à reformuler les phrases pour les rendre conformes à la syntaxe qu'il impose.

Mais la tendance la plus insidieuse de cette traduction reste l'occultation de l'oralité au profit de l'écrit ou d'un essai d'écriture. Ici, l'oralité fait partie intégrante de la signifiance du texte. Tout le texte de Trungpa déploie une sémantique du parlé. Ne pas tenir compte de cette caractéristique du discours de sagesse, c'est en retirer la chaleur, la passion, le souffle. Dans cette traduction, on a voulu rendre le sens, en s'attachant tellement à la lettre qu'on en est arrivé à détourner et la lettre et le sens. On a voulu présenter des idées, un message. II reste des mots sans tessiture, froids, alignés avec maladresse et ayant l'air de porter un contenu.

A quoi pourrait ressembler une traduction de ce même passage si l'on se souciait d'en rendre la signifiance? Voici une possibilité :

ll est bien sûr essenticl de ne pas appuyer la folie des gens même si on les apprécie. On ne peut appuyer aucun type de folie, mème pas la moindre étincelle, même pas sa propre folic. Il n'est pas nécessaire de manquer de lact mais, comme on l'a déja dit, il est possible de travailler sur tout lien qu' une personne crée avec quelque chose, $n$ 'importe quel pesit détail. Essavez de travailler aver la pointe fine de la situation en étant très pratique et ordinaire. l... I Il faut donc garder les pieds sur terre. C'est alors qu' on peut travailler avec les autres.

\section{FILTRAGE REl.igituX ET ANNEXION À LA PHILOSOPHIE OCCIDENTALE}

II faut comprendre que le traducteur occidental qui rend un discours de sagesse dit "oriental» aura parfois tendance à ennoblir la parole du maître parce qu'il perçoit celle-ci comme un discours du sacré, un discours religieux, venant d'en haut. Ce discours embelli produit alors un long contresens : il défait le propos tantrique puisque celui-ci cherche à dire les choses telles qu'elles sont, à appeler un chat, un chat.

Le macrocontexte dans lequel se déroulaient les enseignements de Trungpa doit ici être rappelé. Au début des années 70 , comme il s'adresse le plus souvent à un groupe très hétéroclite d'étudiants, composé en grande partie de hippies et d'autres originaux, il choisit d'employer leur registre de langue, comme le ferait du reste tout bon pédagogue. Par exemple, dans plusieurs des causeries de Trungpa remontant à cette époque, on relève des expressions très vulgaires comme ufuck you", "fucked up", "when you shit and piss". 
etc. $S^{\prime} i l$ avait tenu à ce que ce vocabulaire ne figure pas dans ses textes, il l'aurait sûrement signalé à ses éditeurs. Biffer toute cette richesse langagière qui rend le discours oral percutant, c'est lui plaquer une morale de l'embellissement, de l'ennoblissement ou pratiquer l'ethnocentrisme religieux. Au nom du français correct, on occulte ce qui est perçu comme laid dans le discours parce qu'il houscule les principes fixistes très occidentaux selon lesquels un maître spirituel ne peut employer une langue crue ou carrément vulgaire. L'aspect rugueux, terre-à-terre et immédiat du tantra est dès lors annulé.

Ce type de transformation a des conséquences non négligeables sur l'ensemble d'une ouvre. Prenons par exemple le passage suivant :

Buddha's example is applicable in that Buddha started out basically in the same kind of life that we lead, with the same comfusion. But he renounced that life in order to find the truth. He we'nt through a lot of religious "trips." He tried to neork with the theistic world of hinderism of the time', and he realized there weere a tor of problems with that. The'n. insecad of looking for an ontside solution. he began working on himself He began pulling up his ox"n socks, so to speak. and he became buddha. Until he did that. he was just a wishy washy spirisual ripper.4

Regardons maintenant la traduction donnée de cet extrait et les corrections proposées par une éditrice qui croit fermement à l'élégance et au abon français» ou français de «bon ton». où lè «bon» est érigé comme un principe qui tient de la fixation. En français. on a :

l'exemple du Bondelha s'applique à notre cas en raison du fait qu' il commensa arec le

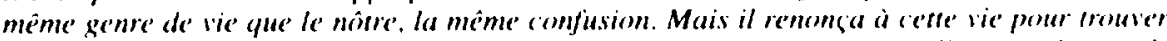

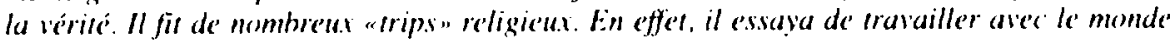
theiste de' l'hindouisme' de l'apoque' et se rendit comple que cela posait beaucoup de problemes. Puis, alu lie'u de chercher ume solution externe, il commenca à sravailler sur lui-

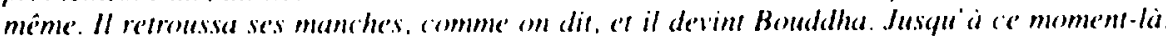

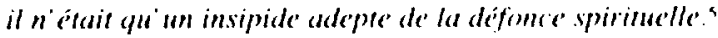

Dans ce texte, l'éditeur veut remplacer le syntagme «trips religieux» par «tentatives religieuses». Cette traduction est fautive à plus d'un titre. D'abord, le mot "trip" ne risque pas d'être mal compris en France : on l'entend, entre autres, au cinéma assè souvent. même s"il ne fait pas «bon ton". Il tigure même dans la dernière édition du Noure'su Petit Robert. Dans la bouche de Trungpa, ce mot a une connotation péjorative. c'est plus qu'une simple tentative, c'est plutôt une démarche un peu illusoire, avortée, une lubie à laquelle on se laisse prendre. En outre. il faut rapprocher cette idée de celle qui est exprimée dans la dernière phrase de l'extrait. Le «wishy washy spiritual tripper". que l’éditrice propose de traduire par «intoxiqué spirituel insipide», renvoie le lecteur à ces «trips religieux». Pourquoi mettre le mot "trips" ? Ici. l'auteur fait justement allusion aux expériences bien connues des hippies de l'époque. Il utilise des images qui les concernent directement. II y a un lien direct entre ces "trips religieux" et l"«insipide adepte de la défonce spirituelle" qu'était, selon Trungpa, le Bouddha à une certaine époque de sa vie. Mettre la jolie expression «tentatives religieuses". c'est détruire ce lien. Le discours de Trungpa est parfois irrévérencieux. Le traducteur n y peut rien : il doit le rendre. Occulter cet aspect de l'ceuvre. cest aussi mettre en cause l'adresse du maître et. à la limite, le caractère compatissant de son activité. Ces attributs du discours montrent le souci que Trungpa avait d'adapter ses enseignements à son auditoire. Il faut donc les faire ressortir comme étant organiques au message transmis.

De plus, confiner le texte de Trungpa à un seul registre de langue (la bonne). e'est supposer qu' un certain niveau de langue serait sacré : c'est mépriser aussi la simplicité, la chaleur humaine qui se dégage d'un discours oral imparfait, avec ses redites. avec son 
affectivité. Si toute l'entreprise tantrique vise à «déconceptualiser» la vie du pratiquant, il serait incongru de la rendre par écrit avec le carcan du beau et de l'élégance, surtout quand celui-ci a tendance à tout ramener à des idées, à des abstractions.

Il arrive, en outre, que le traducteur perplexe devant un système de concepts qui ne lui est pas familier - ou dont il n'a pas une expérience de première main — s'inspire d'une terminologie philosophique occidentale faisant résonner le texte bouddhique sur un mode quelque peu sartien, par exemple.

\section{ORAL CONTRE ECRIT}

Ce qui est mis en cause ici, c'est la propension à faire passer un discours à fonctionnement oral à un texte marqué par les conventions de l'écrit. Le jeu des signifiants est interrompu pour que prime le signifié. Dès lors le discours ne dit plus : il «veut dire». Pareillement, dans la causerie, organisée en texte, le message est inséparable de sa structure, et c'est le rythme pressenti du discours qui tiendra lieu de voix. Escamoter cette exigence c'est traduire le texte sans prendre le temps de le pénétrer à fond, c'est céder à la nervosité du faire. Le discours de sagesse n'est jamais issu de l'agitation mentale. Comme cette parole part d'un point de paix. le traducteur doit accomplir sa tâche depuis le même lieu de "présence attentive" au discours offert. Il doit aussi se détacher du lexique, de la phrase et du sens étroit pour exécuter l'opération de réécriture qu'est la traduction. Il s'agit de choisir entre se conformer au système de la langue ou entrer dans la signifiance du texte pour en dégager «les valeurs propres à un discours et à un seul». Le traducleur s'abandonne ainsi à une danse, à un rythme :

Organisant ensemble la signifiance et la signification du discours, le rythme est l'organisation même du sens dans le discours. Et le sens étant l'activité du sujet de l'énonciation, le rythme est l'organisation du sujet comme discours dans et par son discours. (Meschonnic $1982: 217$ )

Il me semble que ce n'est qu’a cette condition que la vivacité du discours de sagesse peut être transmise. Ce qui suppose que le traducteur aura une expérience concrète, directe. même si elle n'est que partielle, des enseignements qu'il donne à lire. Autrement, il aura toujours tendance à sacraliser le discours, à mettre son auteur sur un piédestal. On ne peut traduire que ce qu'on comprend ou que ce dont on a une intuition. Le traducteur doit être complice du maître, mais plus encore : "Qu'on ne s'y trompe pas : jamais le traducteur ne doit imenter mais il doit toujours trouver, trouver exactement ce qu'a dit l'auteur, il ne doit pas se mettre à sa place, il doit être à sa place.» (Goldschmidt 1986: 162) Ce pari peut sembler impossible pour ce qui est du discours de sagesse. Il reste que, toujours selon la pensée tantrique, c'est justement dans sa pratique du traduire que le traducteur est tenu de transcender son «objection», sa "résistance». Lorsqu il ne comprend pas intellectuellement - qu'il traduise une causerie ou un poème - il lui reste à s’abandonner au discours comme il est, à ces sens qui lui échappent.

\section{TRADUIRE, REECRIRE.}

Dès qu'on pose que l'opération traduisante est bien plus qu'un passage d'une langue à l'autre ayant pour objectif de restituer le sens de l'original, on indique par là qu'elle exige du traducteur une sensibilité d'écrivain. Comment insuffler à un discours un rythme, une authenticité, une organicité textuelle, sans posséder les ressources qu'on attribue généralement à celui qui entre en situation d'écriture comme le signale Barthes :

Cette situation est celle-là même où s'opère un certain ébranlement de la personne, un renversement des anciennes lectures, une secousse du sens. déchiré. exténué jusqu'a son vide 
insubstituable, sans que l'objet cesse jamais d'être signifiant, désirable. L'écriture est en somme. à sa manière, un sutori : le satori (l'événement Zen) est un séisme plus ou moins fort (nullement solennel) qui fait vaciller la connaissance. le sujet : il opère un vide de parole. Et c'est aussi un vide de parole qui constitue l'écriture : c'est de ce vide que partent les traits dont le Zen, dans l'exemption de tout sens, écrit les jardins, les gestes, les maisons, les bouquets, les visages, la violence. (1970: 10)

Le discours de sagesse est issu de ce même vide et de son contraire, la plénitude. C'est dire que cette parole part du silence et non du désir de produire des sens à donner à lire. Le sens se construira au fur et à mesure si le traducteur se fait l'hôte de l'auteur à rendre, au point de devenir cet autre, dans le silence de sa parole. Ce sera même la seule manière de restituer ce que les mots ne disent jamais, peu importe la langue, daller à la rencontre du sens. En d'autres termes, pour entendre le sens, il faut faire silence, c'est alors que le texte - ou tout au moins sa vitalité, son mouvement - est chuchoté peu à peu. Une telle entrée en intimité avec ce qui se construit peut se comparer à une danse où la musique est le verbe de départ et le verbe d'arrivée transcendés.

Mais cela ne veut pas dire que le texte ne fera pas l'objet de multiples retouches ni que le traducteur ne sera jamais en proie aux doutes ni au découragement. Cette danse n'est pas un nirvana ailé. En fait, ce à quoi il faut arriver, c'est à une espèce de dépouillement. car pour le traducteur qui a accompli les exercices tantriques et étudié le tantra. le texte de sagesse tel que parlé par Trungpa est souvent simple, direct, clair. Il n'y a pas de clarté à ajouter. Les passages qui dépassent son entendement doivent rester insondables. C'est alors qu'il doit faire confiance au mot, au rythme de son écriture et non fabriquer du sens. Le texte lui-même suspend la panique qui consiste à hyperconceptualiser la traduction, à lui faire dire ce qu'elle tait. Ce dérapage qui fait parler la société d'accueil du discours un peu trop fort $\mathrm{ct}$, partant. l'annexe. On doit donc apprendre le sens de la mesure et de la démesure. Barthes:

La mesure du langage est ce a quoj l'Occidental est le plus impropre : ce n'est pas qu'il fasse trop long ou trop court, mais toute sa rhétorique lui fait un devoir de disproportionner le signifiant et le signifié, soit en «délayant" le second sous les thots havards du premier, soit en "approfondissant" la forme vers les régions implicites du contenu. (1970: 99)

Cette mesure reste juste tant que le traducteur est apte à revenir au silence, au vide de parole. Tant que le texte de sagesse ne l'effraie pas, e'est-à-dire ne le porte pas à le détoumer de sa vérité rugueuse, choquante. difficile à atteindre. En somme, pour dire la signifiance, il faut, d'une part. rendre ce qui est là et rien de plus selon la manière dont ce qui est là est donné dans le discours original. D autre part. il est essentiel de trouver le courage de remettre en cause ses présupposés sur la traduction. de s'interroger sur leur pertinence, sur leur authenticité. c'est-à-dire de ne pas craindre de critiquer sérieusement son approche du texte. Sans quoi, il s'avère impossible de se mettre en état de réécriture. au service de l'auteur et de son discours. En clair, le traducteur doit se garder d'entrer aveuglément dans l'ordre du système d'écriture - el donc de pensée - établi que fíxe la société d'accueil de la traduction. ("est ce qui faisait dire récemment à Nyssen :

Ainsi a-t-on pu comprendre, par les nouvelles traductions d'André Markowicy, entin attentives au mécanisme génératif de l'écriture, que Dostö̈evski, adversaire déclaré de l'élégance. avait longtemps été lu ici dans un français de honne compagnie qui ne trahissait pas seulement son temperament mais aussi. en quelque sorte. le sens initiatique de ses lextes. $(1993: 47)$

Pour réécrire, il est nécessaire de se sentir libre, de ne pas se conformer, d'inventer. de risquer tout en tenant compte des contraintes inhérentes à la traduction. 
Certains soutiennent qu'il faut faciliter la tâche au lecteur et plaire au ugoût français». Une telle perspective cache une condescendance à l'égard du lecteur ; elle indique aussi que le traducteur hésite à faire le saut dans l'inconnu, dans le vide de la traduction : ce moment suspendu avant que le mot, la phrase, la formulation juste se pose sur l'écran de l'ordinateur. Pour ne pas faire face à l'ébranlement que provoque ce «vide de parole», dont émane le discours de sagesse, le traducteur cherche à produire de la beauté, de l'élégance, de l'exactitude, de la clarté. Il veut son texte concis, efficace et n'hésite pas, ce faisant, à détruire le mode de signifier de l'auteur, qui fait corps avec son discours. Il introduit ainsi un dualisme qui prend sa source dans le vieux rapport fond/forme, signifiant/signifié, langue/énoncé. Tant que le texle de sagesse ne sera pas appréhendé comme un tout organique, dont la signifiance est indissociable du sens proposé, on offrira au lecteur une pensée tronquée, acclimatée. une parole qui sonne faux et dont la force est atténuée. Sa sagesse même, sa raison d'être, sera occultée.

\section{TRADUIRE LA SIGNIFIANCE}

Idéalement, au lieu de ramener le discours à ses présupposés historiques, philosophiques et linguistiques, le traducteur doit se mettre à l'écoute de ce texte perçu comme “autre» et le faire sien, s'y fondre, disparaître dans l'acte de traduire, sortir du dualisme de la langue pour laisser passer la voix de ce discours, sans la filtrer. Il y a lieu de déconceptualiser l'approche de la traduction, de la sortir de l'abstraction intellectualisante à laquelle des siècles «d'écriture» l'ont confinée. C'est le meilleur moyen de ne pas dénaturer un texte pour le faire entrer dans le moule du discours dominant sur la traduction qui expulse la différence - l'auteur en tant qu'il est autre et son mode de signifier - pour céder au dogme de la transparence. C'est la perspective vers laquelle doit tendre le traducteur s'il veut échapper aux tendances annexionnistes qui menacent sa pratique. Dans le cas du discours oral, Meschonnic dit : "L'oralité en effet oppose le rythme et le schéma, c'est-à-dire le mouvement de la parole et de la vie dans le langage, au modèle statique du dualisme, qui ne peut pas comprendre la poésie parce qu il $n^{\circ}$ a rien à voir avec l'empirique du langage, de l'histoire, de la "vie"». (1985: 104) Pour entrer dans ce rythme. le traducteur doit notamment avoir des idées claires sur sa pratique du traduire, établir un rapport direct avec le texte à rendre et le texte à construire, à faire sortir de l'ombre. Pour y parvenir, il est impérieux de savoir faire taire ce bavardage mental, qui le rend sourd au discours déployé. C'est pourquoi il est utile de connaître une expérience pratique et régulière de la méditation, exercice d'attention concret, qui l'amène à ne pas solidifier ni analyser les pensées qui traversent son esprit. La méditation n'est cependant pas une panacée. Elle crée des conditions favorables, mais ne «déconditionne» pas le pratiquant dans des délais record. $C^{\prime}$ 'est pourquoi le traducteur-méditant devra toujours revoir ses préconceptions sur l'art du traduire. Se mettre en jeu : risquer le dialogue avec ses collègues.

De plus, il est évident qu'il doit connaître d'expérience le sujet dont il parle. sans quoi l'implicite du texte, et sa densité. ne peuvent être rendus. En effet, comment traduire le non-dit d'un rituel auquel on n'aurait jamais pris part? C'est souvent lorsqu'il n'a pas l'expérience concrète de ce qu'il traduit que le traducteur met en ceuvre les pratiques annexionnistes dont je viens de parler, pour suppléer.

Rendre la signifiance suppose faire corps avec le discours au point où le texte «est». Car les «modes de signifier sont intimement liés aux modes d’être». (Cordonnier 1992: 190) Le traducteur doit apprendre à transcender ses habitudes d'écriture pour laisser être l'auteur à traduire. Lui servir de pont. Ce qui requiert toutes ses compétences d'être humain, toute son expérience de la vie telle qu'il la ressent, pour ne pas faire écran au 
discours de sagesse. Puisant dans sa longue expérience de praticien de la traduction littéraire, Stratford écrit :

Le traducteur doit plutôt laisser là mots et analyse, se retirer en lui-même et puiser dans sa propre expérience les ressources qui lui permettront de rendre les effets créés par l'original. En d'autres mots, il doit partir à la recherche d'un moment, d'un événement, d'une personne ou d'une voix qui éveille en lui une résonnance - a la fois semblable et différente susceptible de le mettre au diapason du texte (de la tonalité affective, du choix et du registre du vocabulaire). Il ne lui suffit pas de vibrer aux mots et aux pensées de l'auteur ; il lui faut egalement retracer en lui les sources de la vibration et ainsi remonter à l'origine de toute création. (199.3: 125)

Ce qui donne à penser que la traduction du discours de sagesse exige du traducteur qu'il intègre à sa pratique des outils pour se connaître mieux.

\section{CONNAISSANCE DE SOI}

Au fil des années, j’ai observé que les chercheurs spécialistes de la traduction ne traitent presque jamais du traducteur comme être humain agissant sur le texte. Le discours de sagesse oriental, quant à lui, vient faire un peu de lumière sur cette tendance à occulter l'acteur principal du processus de traduction. On voit mal, en effet, comment le traducteur pourrait prétendre à l’intégrité intellectuelle s'il ne décidait pas de réfléchir sur les présupposés qu il nourrit face à l'acte de traduction. On peut se demander pourquoi ce sujet est passé sous silence comme le souligne Annie Brisset :

II est peu question de lui [le traducteur] dans les écrits sur la traduction. Le traducteur est pourtant loin de répondre à l'idéal de transparence, lui-même indissociablement lié à l'objectif de fidélité qui serait inhérent a la visée de l'opération translative. Le discours traduisant révèle un sujet d'énonciation où se croisent des pulsions et des signes idéologiquess qui structurent l'acte de traduire. La psychanalyse s'est déjà penchée sur l'inconscient du traduit. (On a moins vu que le traducteur est aussi un sujet déterminé par l'ordre du discours qui règne dans la seciété dont il se fait le porte-parole. (1990):317-318)

Il me semble qu'une réflexion théorique laissant de côté la question du sujet traduisant, comme s'il était un intouchable, manque une belle occasion d'approfondir la discipline sur laquelle elle pose un regard critique. S'agissant de traduire le discours de sagesse, la question de la connaissance de soi - en tant qu'être humain traducteur — semble évidente.

La traductologie a négligé jusqu ici la question du traducteur comme "être" lecteur du texte et du monde. Or, pour ce qui est de la traduction du discours de sagesse, l'être étant au centre de la question - puisque sagesse signifie savoir être - , il va de soi d'en parler. C'est ainsi que je propose la connaissance de soi comme compétence nouvelle à acquérir. Ce qui veut dire se montrer attentif à sa façon de traduire, mais aussi d'être au monde. dans toutes les siluations de la vie, puisque ce sont elles qui nourrissent l'inspiration du traducteur. Si le travail du traducteur littéraire relève de l'art, il apparâit opportun pour lui de pratiquer un certain art de vivre. une attention qui le tient en éveil, proche du discours qu'il traduit. Le cloisonnement entre l'approche du traduire et la manière de vivre, a bien y réfléchir, $n$ a pas de raison d'être. Si le traducteur vise une perception directe du texte à rendre, il doit s'employer à aiguiser cette faculté d'être dans: l'instant, sans relâche. Il cherche, ne jouons pas sur les mots, à être le discours de sagesse. Son éthique en est une de la présence et commande un déconditionnement linguistique, traductologique, et spirituel. 
Et si l'étude de l'acte de traduction continue de faire abstraction du principal intervenant dans cette opération de passage d'une culture à l'autre, d'une pensée nouvelle parfois déroutante. elle risque de tourner autour du pot, à demeure, pour échafauder des théories qui $n$ 'en finissent plus de faire entendre le même ronron. Je propose, au fond, d'accorder un peu plus de place à l'être humain dans l'étude du traduire. Ce qui rendrait sûrement plus fécond le difficile dialogue entre praticiens et théoriciens. La théorie pure et dure tinit par assécher.

\section{CONCLUSION}

La transmission du discours de sagesse est affaire d'oralité. Depuis toujours. En remontant aux documents anciens qui traitent de l'utilisation de la langue et de la parole depuis Sakyamuni Bouddha, on a vu que le discours de sagesse a toujours été adapté à ses destinataires, que celui-ci avait d'abord une fonction utilitaire. Il s'agissait de faire passer le message, non de l'esthétiser. Par ailleurs, l'examen de traductions récentes de textes de Trungpa montre que le principe du discours oral, direct, qui doit marquer la transmission authentique de ces enseignements, est par trop secoué par des habitudes fixistes bien ancrées. En effet, la tradition française, fortement axée sur l'écrit, tend à faire taire la voix, à la ramener à une construction sentie comme littéraire, amie du beau et de la clarté. J'ai démontré la nécessité de bousculer ces préjugés pour éviter l'écueil de l'ethnocentrisme, refus de la voix (voie) étrangère, et ferment d'exotisation. Au centre de la question. il y a la signifiance, soit le principe de réduction de la dualité entre le discours traduit et son contenu. C'est-à-dire que le discours de sagesse, tel que professé par Trungpa. a son propre mode d'être et que c'est cela même que le traducteur doit chercher à restituer : faire ressortir, dans la réćcriture du texte, les valeurs propres à ce discours et à lui seul. Comment? D'abord, remettre sérieusement en cause ses habitudes et présupposés de traducteur, d'artisan du texte - voir en quoi ils peuvent constituer une barrière bloquant l'expression spontanée. caractéristique de l'oral. De plus, le traducteur doit apprendre à faire silence : une osmose peut dès lors s'opérer entre texte à traduire et traduction. Dans ces conditions, la traduction, comme travail de réécriture. est moins le résultat d'un processus analytique que celui d'un rapport direct, déconceptualisé, avec le texte de départ. En règle générale, le traducteur accorde une importance démesurée au signifié et malmène, dans cet élan, le signifiant. Pour rendre le discours de sagesse dans toute son organicité, il serait avisé de réduire au maximum cette habitude de dualité. Un texte de sagesse est percutant par sa présence même, son unicité. Pour opérer cette unicité du texte, l'esprit du traducteur doit connaître le repos, le calme, une trêve de conceptualisation. La traduction exige de l'exécutant au moins quatre grandes qualités associées à l'état mental dans lequel il travaille : attention, précision, acuité, capacité de concentration. L'exercice de la méditation. pratique fondamentale du tantra, cultive ces qualités.

Traduire le discours de sagesse m'a appris qu'il fallait faire entrer la «vie» au cour de la réflexion théorique. J'espère que les chercheurs en traduction oseront de plus en plus faire état d'expériences plus personnelles, intimes. La sagesse de l'expérience aidant, il n'est pas impensable que de la réflexion traductologique se dégage un jour davantage de chaleur humaine, qu'une compassion éblouisse le discours, l'éclaire tout au moins. L'audace, la folie manquent dans les cercles traductologiques. J'arrête là cette conclusion. avant de passer pour un «illuminé». Le risque est réel quand on travaille des textes bouddhiques : le mot sanskrit Buddha est parfois traduit par l'Illuminé. Je sais toutefois que cette lumière, quand elle n'est la propriété de personne, traverse tranquille, douce et chaude. Qu'elle entre donc vivement comme une musique qui fait sourire jusqu'aux larmes! 


\section{Notes}

1. Nom des textes religieux et peétiques qui forment les premiers documents littéraires de l'Inde. ecrits en sanskrit archaïque.

2. Revue (Mharma, n" 8. Institut Karma-l.ing. p. 57.1990

3. Naropa Instisute Jomernal of Pswhology. vol. 2. Nalanda Press. Boulder. 198.3, p. 1.

4. "Taking Refuge". C. Trungpa, Caruda V. Boulder, Vajradhatu et Shambhala Publications, 1977. p. 20

5. "Chercher refuge», S, Bédard, 1991 .

\section{RÉFERENCES}

BARTHES. Roland (1970) : l'enpire' de's signe's, Paris, Flammarion.

BARTHES. Roland (1984): Le hruissement de la langue', Paris, Seuil.

BERMAN, Antoine (1982) : "La traduction des auves latino-américaines», Lendemains, $n^{\circ} 27$. Berlin. pp. $39-44$

BERMAN, Antoine (1986) : "L'essence platonicienne de la traduction", Reviue d' ésthértqué. nouvelle série n' 12. Editions Privol. pp. 6.3-7.5.

BRISSET, Annie (1990): Socierritique de la rraduction, Québec, collection de l'Univers du discours, le Préambule:

CORDONNIER, Jean-Louis (1992): «1492-1992 Voyage sur la mer océane: traduire et dévoiler", Mr'a. Montréal, PUM, vol. 37, $n^{\circ} 2$, juin, pp. 174.192

DERRIDA, Jacques (1967) : De la (irammatologie, Paris, Editions de Minuit.

GOLDSCHMIDT, Georges-Arthur (1986) : “Écriture, Iraduction, liberté", Rerue d'esthérique, nouvelle série $n^{\circ} 12$, Editions Privot, pp. 159-164.

LAMOTTE, Etienne (1976) : Histoire du houddhisme indien, Louvain, Institut orientaliste Louvain-la-Neuve.

MESCHONNIC. Henri (1982): Critique du rythme. Paris, Editions Verdier.

MESCHONNIC. Henri (1985) : Le's éfats de la poétique. Paris, PUF.

MESCHONNIC, Henri (1986): "Alors la traduction chantera", Rev'ue d'esthétique, nouvelle série n" 12. Éditions Privot, pp. 75-90.

NYSSEN, Hubert (1993) : “De la traduction”, Liberté. Montréal, vol. 35. n 1. pp. 44-.56.

SCHLECHTEN, Marguerite (1987) : "Oralite". Ethologica Heliefica. vol. 11. Société suisse d"ethnologie

STEINER, George (1977) : After Bahol, London. Oxford University Press.

STRATFORD, Philip (1993) : "L écrivain clandestin", Liberté. Montréal, vol, 35, n 1, pp. 120-131.

TRUNGPA. (högyam (1993) : Folle sagesse', trad. par Zéno Bianu, Paris, Éditions du Seuil. 